\title{
Analysis of refill curve shape in ultrasound contrast agent studies
}

\author{
T. C. Potdevin, ${ }^{\text {a) }}$ J. B. Fowlkes, A. P. Moskalik, and P. L. Carson \\ Department of Radiology, University of Michigan, Ann Arbor, Michigan 48109-0553
}

(Received 8 October 2003; revised 18 December 2003; accepted for publication 18 December 2003; published 26 February 2004)

\begin{abstract}
Contrast destruction and replenishment by Flash Echo Imaging (FEI) (also referred to as interval or intermittent imaging) has been qualitatively and quantitatively used for tissue blood refill measurements. Many features and capabilities of contrast refill in tissue blood flow and perfusion remain to be elucidated. To aid the development and full reliable utilization of the technique in medical practice, in this paper we undertake physical and mathematical modeling to evaluate different measures derivable from FEI and to provide a basis for the further study of sensitivity and stability of such measures for the detection and measurement of various flow properties and abnormalities. A phantom was developed and used to conduct a dynamic contrast study. Refill curves were investigated as a means of calculating the mean transit time (MTT) and investigating other information that can be determined from their shape. Exponential and error function fits and the area above these curves were used to estimate MTT. The bubble disruption zone was visually measured and theoretically modeled. Computer simulated refill curves based on the flow phantom for different velocity ranges were then computed and compared to the experimental refill curves. The simulated refill curves closely matched the experimental curves in both shape and MTT. The simulated refill curves matched the shape of the experimental results for different velocity ranges. Another simulation examined how a real circulatory system might influence refill. Different refill curve shapes were obtained for different vascular models. Models including the large arteries and veins showed a much faster initial slope than models where the large vessels were not included. Likewise, simulated "shunting" displayed a different slope than models without "shunting" and specific portions of the refill curve that could maximally distinguish shunting. This computer simulation could lead to some experimental hypotheses about differences between normal and cancerous blood flow. () 2004 American Association of Physicists in Medicine. [DOI: 10.1118/1.1649534]
\end{abstract}

Key words: medical imaging, diagnosis, mean transit time, perfusion, simulation

\section{INTRODUCTION}

For about half a century, indicator-dilution theories for volume flow measurement have been investigated. The indicator-dilution theory was first developed for cardiac output measurement ${ }^{1-4}$ and has now been used for blood flow measurement. However, several problems with practical use were encountered such as the necessity of two catheters for controlled indicator release and for the region of interest (ROI) sample withdrawal, some anatomical regions cannot be reached for catheter use. In addition, contrast agent must be released in the left ventricle for uniform mixing in the blood, and the timing for both contrast agent release and blood withdrawal is critical.

Ultrasound flash echo imaging (FEI) offers a powerful alternative because it is minimally invasive, inexpensive and overcomes the problems mentioned above. $\mathrm{FEI}^{5,6}$ or intermittent imaging ${ }^{7}$ has been qualitatively and quantitatively used for tissue refilling measurements. For instance, the mean transit time (MTT) of a tissue volume can be estimated. ${ }^{3,4}$ For our purposes, MTT is defined as the average time that flowing particles take to exit from anywhere within a given volume. In FEI, the measurement technique consists of destroying microbubbles in tissues using a high mechanical index (MI) ultrasound (US) beam and monitoring the tissue refill with different time delays between destructive pulses. Each destructive pulse is also a monitoring pulse. Alternatively a nondestructive monitor can be used on some US systems to measure the refill.

In this study, a flow phantom was developed to further investigate the information contrast refill can provide about fluid flow. The flow phantom consists of a dialysis tube embedded in tissue mimicking foam. Interchangeable foam inserts were placed within the tube to perturb the parabolic flow profile usually found in an empty tube and to offer some backscattering. The foam inserts were chosen to closely match real tissue with regard to a sound speed and attenuation coefficient. The contrast solution was pumped through a recirculating system at a slow, constant rate.

We observed refill curves that were often more complex than a simple exponential and therefore required further correction to relate the measurement to the MTT. There was often a delay before significant refill occurs due to the bubble disruption zone being wider than the imaging sensitivity zone. Other factors affecting the refill curve shape include the distribution of velocities within the ROI and the point spread function (PSF) of the imaging system sampling the ROI. 


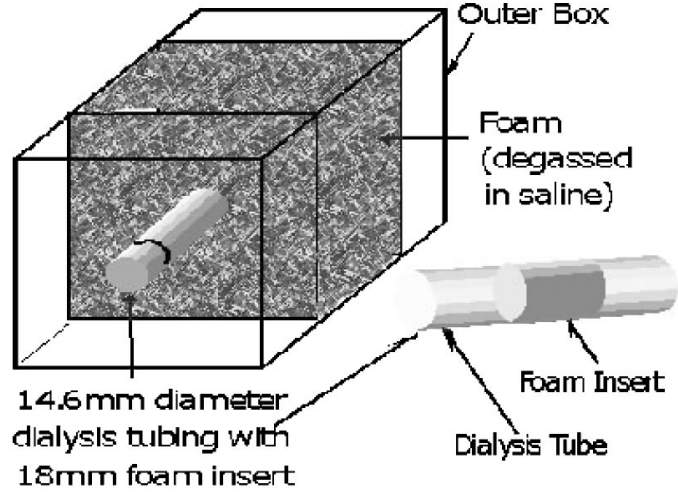

FIG. 1. The experimental setup consisted of dialysis tube through which various types reticulated foam plugs were inserted. A solution of contrast agent and saline was pumped through the system using a recirculating pump system. A block of reticulated foam was used to simulate surrounding tissue. The foam materials were degassed in a vacuum chamber to remove any air bubbles and then immersed in a degassed saline solution contained in a box.

To gain a greater understanding of the factors that affect the refill curves, computer simulations were performed. Simulated curves that closely resemble the actual in vitro studies were generated. In addition, it was possible to investigate a broader range of velocity distributions than in our simple ultrasound flow phantom. For instance, excluding the effects of the imaging PSF, a purely monotonic velocity profile results in a nearly linear refill curve while a parabolic flow profile results in a much steeper initial slope to the curve than an exponential. Another area investigated was the effect of the shape of the bubble disruption zone on the refill curve. These simulations could be useful in developing hypotheses about measurements of in vivo vascular systems for applications such as the detection of cancer.

\section{METHODS}

\section{A. Experimental setup}

The experimental setup consisted of a $14.6 \mathrm{~mm}$ diameter dialysis tube (Spectra/Por molecularporous, Inc., Rancho Dominguez, CA) through which various types of $3 \mathrm{~cm}$ long reticulated foam plugs (either S110, S80, T50 or A40) (Crest Foam Industries, Inc., Moonachie, NJ) were inserted (Fig. 1). The void volume was $97 \%$ and the uncompressed diameter was $18 \mathrm{~mm}$. A solution of contrast agent (MRX-133 or 408, ImaRX Therapeutics, Inc., Tucson, AZ) and saline (1000 ml of $0.9 \%$ sodium chloride, injection, USP, Abbott Laboratories, North Chicago, IL) was pumped through the system using a recirculating pump (variable pressure volumetric pump, IVAC Corporation, San Diego, CA). The contrast agent used is a suspension of lipid stabilized perfluorocarbon microbubbles. This type of contrast agent has a relatively low buoyancy and therefore a very slow rise time. There was no apparent marginalization of the contrast agent in the flow tubes to and from the phantom.

A block of reticulated foam (S110, Crest Foam, Inc.) was used to simulate the surrounding tissue. The foam materials were degassed in a vacuum chamber (Welch duo-seal vacuum pump, Sargent-Welch Scientific Co., Skokie, IL) to remove any air bubbles and then immersed in a degassed saline solution contained in a box.

\section{B. Acoustic properties}

Each foam insert was evaluated for basic acoustic properties by placing each sample in degassed saline between a matched pair of transmitting and receiving transducers $(\mathrm{KB}$ alpha 3.5 MHz H 30871, K. B. Aerotech, Lewiston, PA). A pulser/receiver system (Panametrics, Model 5900PR, Waltham, MA) produced narrow band pulses and an oscilloscope (Hewlett Packard Infinium, Colorado Springs, CO) recorded the signal from the receiving transducer.

The ultrasonic pulse's transit time was used to calculate each foam plug's speed of sound for a $2 \mathrm{~cm}$ saline path at room temperature $\left(\sim 23^{\circ} \mathrm{C}-24^{\circ} \mathrm{C}\right)$. The power spectrum of the pulse was measured before and after insertion of the foam plug in the ultrasonic beam in order to compute the attenuation coefficient in $\mathrm{dB} / \mathrm{cm} / \mathrm{MHz}$. This was calculated by taking the slope of the difference of these two power spectra.

\section{Refill curve acquisition}

A Toshiba Power Vision 8000 scanner (Toshiba American Medical Systems, Tustin, CA) was employed for imaging, with a $3.75 \mathrm{MHz}$ curvilinear probe transmitting at $2.3 \mathrm{MHz}$ in the contrast FEI mode. The combination dialysis tubefoam insert was positioned at the elevational beam focus and viewed in a cross-sectional plane, i.e., an imaging plane perpendicular to the flow direction. The focus was measured using a slice profiling phantom to be $5 \mathrm{~cm}$ in the FEI mode.

A 1/5000 diluted contrast agent in a saline solution was circulated in the closed system described above. Refill curves were evaluated for four reticulated foam inserts whose pore size and density differ from each other and at three different flow rates $(156,312$ and $624 \mathrm{ml} /$ hour). Although these flow rates were slow, due to the contrast agent's slow rise time, the signal level remained constant throughout the experiment indicating that minimal or no contrast agent separation occurred.

In the contrast FEI mode, the images were captured with the system operating solely in the high power mode, i.e., with the scanner's monitor feature turned off. Fourteen transmitted images and a subtraction frame (subtraction of the 14th frame from the first) constituted each flash sequence with the scanner's acoustic power at its maximum for the largest possible destruction of the contrast bubbles in the image plane of the probe (Fig. 2).

Subsequent images for each flash sequence were acquired after an interval of PI (pulse interval) seconds from the flash. One cine loop of images was acquired for each PI. This yielded 5 flash sequences. This procedure was repeated for monotonically increasing PI between flash sequences for each foam insert and for each flow rate. This entire procedure was also repeated twice for each flow rate and type of foam insert. 


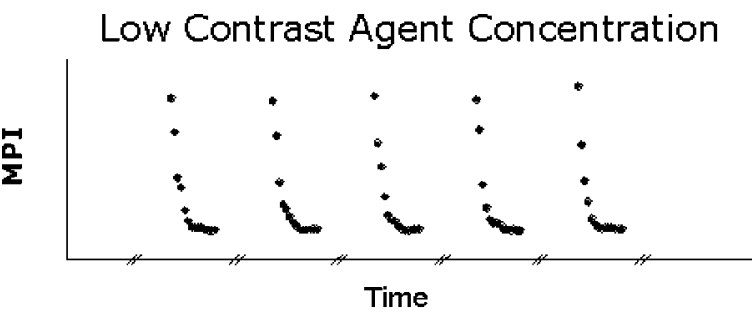

High Contrast Agent Concentration (Veil Effect)

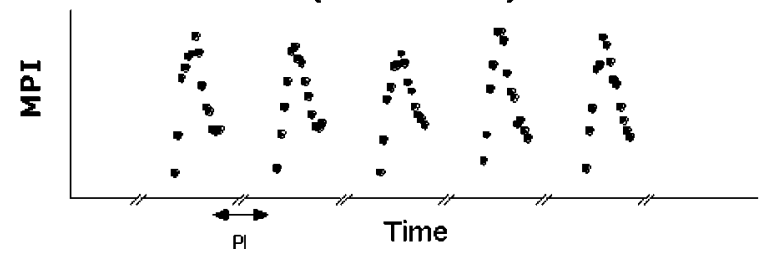

FIG. 2. In an FEI sequence, a series of images were acquired at full frame rate after an interval of PI (pulse interval) seconds. The first series of images removes the contrast signal and each subsequent series measures the contrast refill. This sequence was repeated $N$ times at interval PI. The procedure was repeated with monotonically increasing PI between flash sequences in each trial. This figure represents the mean pixel intensity (MPI) within the region of interest (ROI) of the reticulated foam insert vs frame number for a low and a high contrast agent concentration. The high contrast agent concentration suffers from the veil effect resulting in a low MPI for the initial pulses due to shadowing.

All the acquired images were stored on the scanner's hard disk, transferred to a computer station and then processed. The images were processed using an analysis program written in MATLAB (MathWorks, Inc., Natick, MA). A region of interest (ROI) was drawn onto the foam insert cross-section. The pixel values recorded by the scanner were decompressed to obtain the relative backscattered intensity. The reconstruction of the refill curves, from which MTT can be estimated, was produced by computing a mean linearized intensity values over this ROI (Mean Pixel Intensity, or MPI) for each pulsing interval $(0.5,0.7,1,1.5,3,5,7,10,15,20,30 \mathrm{sec})$. The MPI for each pulse interval was computed by averaging the brightest frame for each of the 5 pulse sequences contained in a cine loop.

\section{Maximum projection}

Often the concentration of the contrast agent in the foam insert created a shadowing effect called the veil effect (Fig. 2). ${ }^{8}$ This effect was characterized by the first frame containing some bright pixels at the top of the ROI while pixels below were shadowed. In subsequent frames, the contrast agent at the top of the ROI was destroyed revealing bright pixels below them. The shadowed pixels cause a low MPI over the ROI. To correct this, a maximum projection technique was used which gives a better indication of the presence of contrast throughout the ROI. ${ }^{9}$

The maximum projection program was also written in MATLAB. The image sequence was cropped to the size of the ROI. For each flash sequence, the maximum pixel value was selected for each $(y, z)$ position across the 14 frames acquired in each flash sequence. The resulting image was decompressed and then the average of the 5 resulting maximum projected images was computed. These maximum projection results were then used to compute the MPI as described above.

\section{E. MTT estimation}

Two methods have been used to estimate MTT. The Meier and Zierler ${ }^{2}$ integration method estimates a particular MTT as the integrated area above the refill curve. Wei's method ${ }^{3,4}$ estimates MTT as the slope of the refill curve at $t=0$, i.e., $1 / a$ when the refill curve is represented by the equation $y$ $=b(1-\exp (-a t))$ with $b=1$. Another technique is to fit the refill curve with the error function $y=\operatorname{erf}(a t)$. In this case, the area above the curve is $1 / a \sqrt{\pi}$ (see the Appendix). Neither fit represented a physical explanation of how the refill should progress, but rather they serve as a way to smooth the raw experimental data.

Ideally, an indicator refill curve with respect to time would initially start at some time 0 then monotonically increase reaching a saturation concentration. Therefore, the indicator concentration was normalized by the saturation concentration. In this study, each raw refill curve was first adjusted by subtracting the initial echogenicity of the foam insert before contrast traverses it and then normalized by the maximum mean pixel intensity over the refill curve. Likewise, the time axis was shifted to account for a delay time (DT) (discussed later) such that $0 \mathrm{sec}$ was defined as the time when the MPI began to increase.

To obtain a single representative refill curve for each flow rate and foam insert, the two associated normalized refill curves were averaged into one single curve and renormalized by the maximum MPI. This curve was then fit by $y_{2}=(1$ $-\exp (-a t))$ or $y_{2}=\operatorname{erf}(a t)$ where, respectively, $1 / a$ or $1 / a \sqrt{\pi}$ represents the estimated MTT for each flow rate and foam insert.

\section{F. Bubble disruption zone and read-out zone}

To evaluate the accuracy of our refill MTT estimation, we also estimated MTT geometrically. To do this, we assumed a plug flow, i.e., a constant velocity across the vessel and then we measured the cross-sectional area, the bubble disruption zone width and the volume flow rate. To measure the bubble disruption zone width, the following experiment was performed.

The same flow phantom as previously described was used with the transducer orthogonally positioned to the flow direction and the flow of diluted contrast halted. The contrast FEI mode with subtraction mode on was used to create a bubble disruption zone using a single set of 15 high MI images as in the refill curve experiment. The transducer was then rapidly rotated through an angle of $90^{\circ}$ to observe the bubble disruption zone using a second set of 15 high MI longitudinal images of the center of the flow tube. The 15th frame of the second set was the subtraction frame, i.e., the subtraction between frame 1 and 14 of the second set. This image was used to measure the extent of the bubble destruction, i.e., the profiles of the flow tube's anechoic bubble dis- 


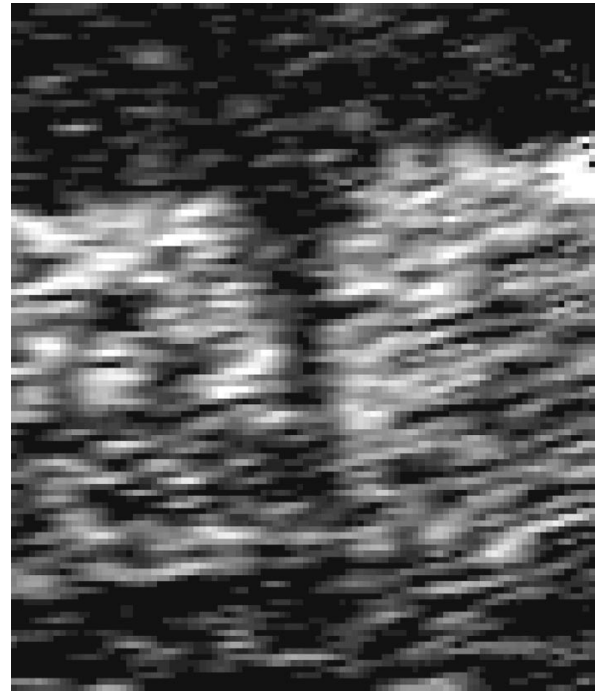

FIG. 3. This subtraction FEI mode image was generated with the transducer longitudinal to the tube after a sequence of high MI pulses in the orthogonal plane. The bubble disruption zone is clearly visible and its width is shown to vary with depth.

ruption zone (Fig. 3). Note that the width of the destruction zone varies with depth through the tube. This effect will be included in a subsequently described model as an initial correction for this effect.

The average width of the read-out zone width can also be estimated by calculating the slope of the velocity versus the inverse of the estimated MTT. The velocity profile in the foam insert was assumed to be constant and was calculated as the volume flow rate (based on the volume output from the fluid pump) divided by the cross-sectional area of the tube as measured on the US image. The area of the foam material itself was considered negligible because the void volume was $97 \%$.

It was observed that the bubble disruption zone was larger than the read-out zone, which was manifested as a delay time (DT) measured as the time between the bubble disruption event and the beginning of the refill rise.

\section{G. Simulation}

The initial computer simulation generated refill curves based on the geometry and assumed properties of the in vitro flow phantom for different velocity ranges. These curves were compared to the experimental refill curves. The simulated tube model consisted of a cylindrical region of radius 50 pixels (Fig. 4). The effort here is to provide some initial corrections for the effects of beam shape and overlying attenuation including that from the presence of the contrast agent and its dynamic changes during FEI.

Each pixel $i$ was associated with a velocity $v_{i}$ and two widths: one related to the bubble disruption zone length (KZ) and the other related to the read-out zone length (RO). The bubble disruption zone's width varies with $z$ and therefore $\mathrm{KZ}$ was calculated at $M$ points along the axial axis $z$. For the purpose of this modeling, this elevational variation was defined as a truncated Gaussian shape [Fig. 4(a)]. In addition, (a)
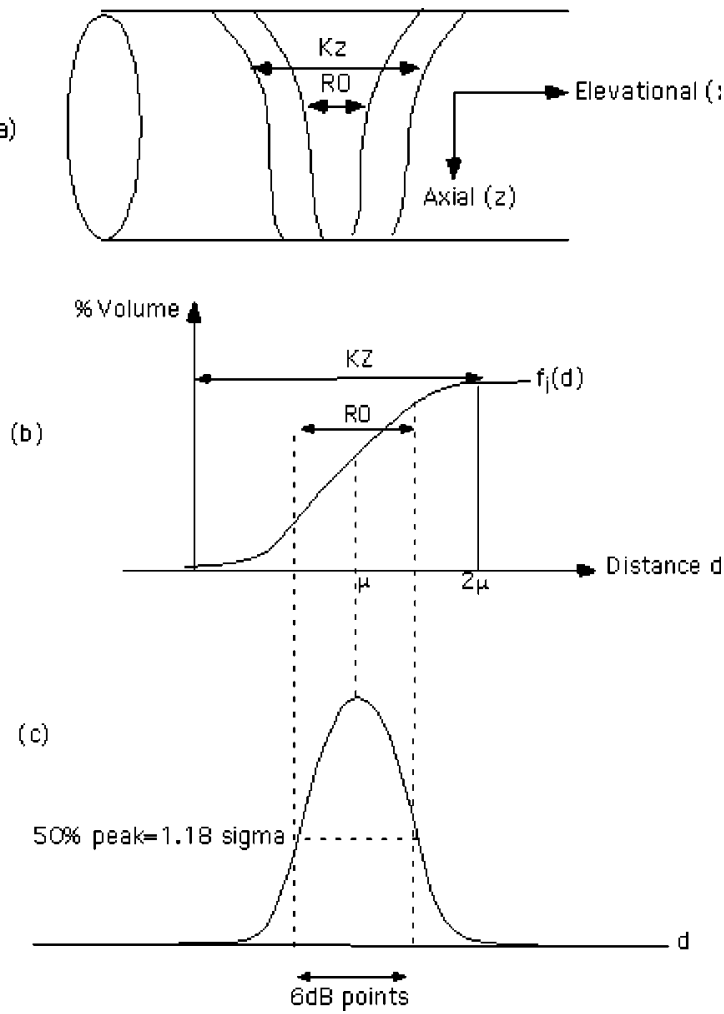

FIG. 4. Generating finite element refill curves. The top drawing illustrates the variation with depth of the width of the bubble disruption (KZ) and the read-out (RO) zones. Often $\mathrm{KZ}$ was wider than RO. Each pixel element $i$ was assigned a KZ and a RO from the relevant depth $(z)$ as well as a velocity $v_{i}$. An elemental refill curve $f_{i}(d)$ is shown here in the middle illustration. $d$ was the distance traveled by the leading edge of the contrast as it refills the bubble disruption zone for this element. $d$ can be simply related to time by the equation $d=v_{i} t . f$ was the cumulative distribution function constructed from a Gaussian shaped point spread function (PSF), shown in the lowest drawing, where the Gaussian means are $\mu=\mathrm{KZ}(z) / 2$ and the standard deviations are $\sigma=\operatorname{RO}(z) / 2.36$.

the read-out zone was modeled as a typical Gaussian weighted point spread function (PSF). Like KZ, the width of this PSF varies with depth $z$ due to attenuation effects.

For each pixel $i$ in the $y, z$ plane, an unnormalized spatial elemental refill curve is generated $f_{i}(d)$, where $d$ was the distance traveled by the leading edge of the contrast through the bubble disruption zone for this element:

$f_{i}(d)=(1 / 2)[1+\operatorname{erf}((d-\mu) / \sigma \sqrt{2})]$ which is the cumulative distribution function due to the Gaussian shaped point spread function (PSF) of the read-out zone with $\sigma$ $=\mathrm{RO}(z) / 2.36$ and the width of the bubble disruption zone with $\mu=\mathrm{KZ}(z) / 2$.

A normalized spatial refill curve can be represented by the equation

$$
F_{i}(d)=\frac{f_{i}(d)-f_{i}(0)}{f_{i}(2 \mu)-f_{i}(0)} .
$$

The velocity was assumed to be uniform plug flow when simulating the foam insert, i.e., the velocity was equal to a single linear velocity for every pixel. When simulating an empty tube, a parabolic velocity profile was employed. In 
TABLE I. Flow properties of the human systemic circulation (Ref. 12).

\begin{tabular}{lcc}
\hline \hline Vascular subset $(j)$ & Velocity $(\mathrm{cm} / \mathrm{sec})$ & Length $(\mathrm{cm})$ \\
\hline Large arteries (1) & 20 & 100 \\
Small arteries (2) & 4 & 13 \\
Arterioles (3) & 2 & 3 \\
Capillaries (4) & 0.03 & 0.1 \\
Venules (5) & 0.3 & 0.4 \\
Small veins (6) & 1 & 10 \\
Large veins (7) & 4 & 100 \\
\hline \hline
\end{tabular}

either case, for each pixel $i, d=v_{i} t$, where $v_{i}$ is the velocity associated with that element $i$.

$F_{i}\left(v_{i} t\right)$ is then evaluated for each pixel $i$ to generate a refill curve with respect to time. The complete refill curve is an average of all the elemental refill curves:

$$
R(t)=\frac{\sum_{i=1}^{n} F_{i}\left(v_{i} t\right)}{n} .
$$

\section{H. Extension to vascular flow}

However, the simulated tube model does not accurately represent a real human circulatory system. Another simulation investigated how a circulatory system with many different sized vessels distributed throughout the imaged tissue might affect contrast refill.

Unlike in the tube simulation model, each finite element was not assigned a single velocity. Rather, a continuous range of velocities was simulated representing the transition between vessel types. The circulatory simulation consisted of a series of large arteries going into small arteries, then arterioles, then capillaries, then venules, then small veins and finally large veins (Table I).

An elemental refill curve was constructed from two functions. The first related time with the distance traveled across the bubble disruption zone $d_{k}=g_{k}(T)$. And the second is the normalized spatial refill curve that was developed in the first simulation.

As in the tube model, the contrast refill was assumed to progress from one side of the bubble disruption zone to the other. However, in this case, a spatial variation along the $z$-axis was included that provided for differing vascular contributions. The assumption is that the image plane being refilled has a finite slice thickness that is small compared to the axial and lateral extent of the plane. Therefore, all flow contributions are from out of plane and can be from either direction. At any given location in the plane, the vasculature would have varying contributions for different vessel types. In addition, as the thickness increases, the distribution would change. For example, capillary beds near the center of the slice must have supply arteries and draining veins. Arterial and venous effects then depend on the location of the capillary bed within the slice.

This function was constructed as follows. The entire circulation path was laid out on the time axis: $t_{j}=l_{j} / v_{j}$, where $l_{j}$ and $v_{j}$ are, respectively, the length and velocity of each vascular subset $j$ for $j=1$ to 7 . In other words, blood spends a $t_{j}$ amount of time in the vascular subset $j$. The total amount of time blood will circulate through the entire system is $T=\sum_{j=1}^{7} t_{j}$. When divided into $N$ equal time intervals, $\Delta t=T / N$.

Within each time interval $\Delta t$, blood flows with a velocity of $v_{l}$ where $l=1$ to $N$. $v_{l}$ is defined by linearly interpolating the velocities associated with the vascular subsets $j$ over the $N$ time intervals $\Delta t$. Points beyond the time point $N$ are assigned the value $v_{N}$. The distance, $\Delta d_{l}$, that blood traverses in $\Delta t$ with a velocity of $v_{l}$ is therefore $\Delta d_{l}$ $=v_{l} \Delta t$.

It is assumed here that for each point in the circulatory system, the volume flow rate is constant. The blood volume associated with a segment of the circulatory system is directly proportional to the amount of time blood takes to traverse it. For each segment of $\Delta t$ length in the vasculature, one expects to have an equal volume of blood associated with that segment. Therefore, in any given sub-volume of tissue in the body, a first order approximation would be that an equal blood volume is input from every $\Delta t$ segment, assuming the different elements of the circulatory system are evenly mixed. The total refill curve would be an average of the refill curves beginning at each $\Delta t$ sub-volume.

An elemental refill curve starts at a sub-volume $l$ of the circulatory system. So we have $N$ elemental refill curves. Each refill curve starts at one edge of the bubble disruption zone and is therefore $\mathrm{KZ}(k)$ in width where $k=1$ to $M$ and represents the depth. Like in the tube simulation, each elemental refill curve is spatially independent and there are $M$ values of $\mathrm{KZ}(k)$. Unlike in the tube model, the velocity can vary with time.

The first step is to generate a function relating the distance traveled with time. This function starts at 0 and reaches a maximum at KZ. Each time segment $\Delta \mathrm{t}$ was assigned a velocity $v_{m}$ for $m=l$ to $N$ which determines the slope of the refill at that time interval. When the distance traveled $d_{k}$ is bigger than $\mathrm{KZ}$ or in other words when the sum of all time intervals traversed $\Delta t$ multiplied by their appropriate velocity $v_{k}$ equaled the bubble disruption zone width (KZ), it reaches saturation and is set equal to $\mathrm{KZ}$. Therefore

$$
\mathrm{KZ}=\sum_{k=l}^{N} v_{k} \Delta t_{k} .
$$

As in the tube simulation, the read-out zone was a typical Gaussian weighted PSF whose width varies with depth due primarily to attenuation effects. The width of the bubble disruption zone also varied with depth. This variation was modeled as a Gaussian shape in this study.

Next, the distance traveled $d_{k}=g_{k}(T)$ was related to the percent refill by evaluating the cumulative distribution function, similarly defined in the tube model. Having $M^{*} N$ values of $g_{k}(T)$, one for each elemental refill curve at each depth, we applied the effects of the $\mathrm{KZ}$ and RO, $F_{k}\left(g_{k}(T)\right)$, as we did in the tube simulation to create elemental refill curves.

The final refill curve is a weighted average of all these elemental refill curves where the weighting for each elemen- 
TABLE II. Reticulated foam inserts' characteristics. The speed of sound and attenuation coefficient of the reticulated foam inserts were measured in a degassed saline solution. The pore size was obtained from Crest Foam Ind. Three methods were used to estimate MTT or the area above the refill curve: the trapezoid method, an exponential fit and an erf fit. The read-out zone length can be estimated from the slope of the pump's volume flow rate normalized by the area of the reticulated foam insert $(\mathrm{cm} / \mathrm{sec})$ vs $1 / \mathrm{MTT}\left(\mathrm{sec}^{-1}\right)$.

\begin{tabular}{|c|c|c|c|c|c|c|c|}
\hline & $\begin{array}{l}\text { Sound } \\
\text { Speed } \\
(\mathrm{m} / \mathrm{sec})\end{array}$ & $\begin{array}{l}\text { Attenuation } \\
\text { coefficient } \\
(\mathrm{dB} / \mathrm{MHz} / \mathrm{cm})\end{array}$ & $\begin{array}{c}\text { Pores/inch } \\
\text { ppi }\end{array}$ & $\begin{array}{c}\text { Density } \\
\text { lb./cu.Ft. }\end{array}$ & $\begin{array}{c}\text { RO width } \\
\text { Vel.*MTT } \\
\text { Area ab. } \\
\text { Curve } \\
\text { (cm) }\end{array}$ & $\begin{array}{c}\text { RO width } \\
\text { Vel.*MTT } \\
\text { Exp. Fit } \\
\text { (cm) }\end{array}$ & $\begin{array}{c}\text { RO width } \\
\text { Vel.*MTT } \\
\text { Erf Fit } \\
(\mathrm{cm})\end{array}$ \\
\hline $\begin{array}{l}\text { Empty } \\
\text { tube }\end{array}$ & 1492 & & & & $0.23 \pm 0.01$ & $0.21 \pm 0.03$ & $0.18 \pm 0.03$ \\
\hline S110 & 1498 & 0.79 & 94 & 2.2 & $0.18 \pm 0.02$ & $0.15 \pm 0.03$ & $0.13 \pm 0.03$ \\
\hline S 80 & 1498 & 0.81 & 80 & 1.9 & $0.14 \pm 0.04$ & $0.12 \pm 0.04$ & $0.11 \pm 0.03$ \\
\hline $\mathrm{T} 50$ & 1496 & 0.75 & 50 & 1.4 & $0.16 \pm 0.07$ & $0.13 \pm 0.04$ & $0.12 \pm 0.04$ \\
\hline $\mathrm{A} 40$ & 1497 & 0.70 & 40 & 1.3 & $0.13 \pm 0.03$ & $0.11 \pm 0.03$ & $0.10 \pm 0.03$ \\
\hline
\end{tabular}

tal refill curve is its blood volume within the slice thickness. This weighting is therefore based on the amount of time blood takes to get through the bubble disruption zone. In other words, a slow refill curve was weighted more because more blood has time to flow into the vasculature associated with that refill curve. Therefore, each refill curve is weighted by its rise time $\mathrm{RT}_{l, k}$ :

$$
R(T)=\frac{1}{N} \sum_{k=1}^{M} \sum_{l=1}^{N} F_{k}\left(g_{l, k}(T)\right) \frac{\mathrm{RT}_{l, k}}{\sum_{k=1}^{M} \sum_{l=1}^{N} \mathrm{RT}_{l, k}} .
$$

This model can easily be adjusted to accommodate different tissue models. For instance, if no large vessels are present in the ROI then they can be removed from the list of vascular subsets. Shunting is characterized by a direct flow of blood from the arterial to the venous system, therefore, can be simulated by bypassing the capillary beds. The shunting simulation was similar to the one mentioned above but omitting the capillaries.

\section{RESULTS}

\section{A. Foam properties}

The speed of sound and attenuation coefficient measured for each foam are presented in Table II along with the porosity and the density. Note that the measured sound speed in the tube filled with only saline matches measurements made within the foam inserts to within $1 \%$. The attenuation coefficient only varies from $0.70-0.81 \mathrm{~dB} \mathrm{~cm}^{-1} \mathrm{MHz}^{-1}$ for the foams selected. Using ROIs selected from the S110 foam block surrounding the tube, the signal to noise ratio was estimated from the decompressed pixel intensities as MPI/ stdev, where the stdev is the standard deviation of the pixel distribution. The resulting value was 1.5.

To ensure that no bubble accumulation occurred during contrast circulation, the echogenicity below the reticulated foam insert was measured and remained constant throughout the experiments.

\section{B. Maximum projection}

The relationship between MPI and the contrast agent concentration is presented in Fig. 5. Due to the creation of shadowing at a high concentration, a decrease in MPI was observed when the concentration exceeded $0.1 \mathrm{ml}$ of contrast in 1 liter of saline. After a correction for shadowing by a maximum projection technique, a linear relationship between MPI and the contrast agent concentration was observed. Therefore, this technique was also used to generate the refill curves.

\section{Refill curve fitting}

Each refill curve was fit with both the error function (erf) and an exponential. The error function was a better fit than the exponential to the experimental curves observed. In the

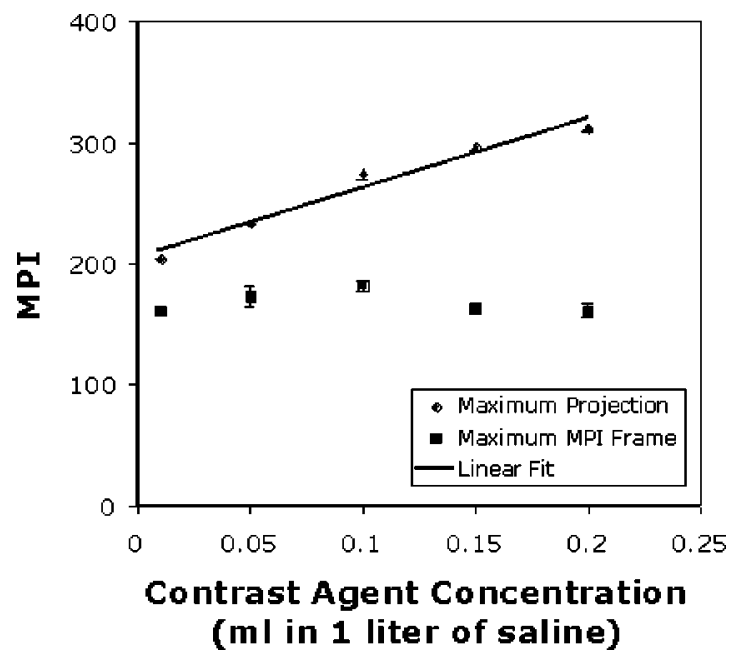

FIG. 5. Relationship between MPI and the contrast agent concentration. The points constituting the Maximum MPI Frame were obtained from the average of the 5 peak frames of a FEI sequence taken in a tube with a S110 foam insert. At high concentration, a shadowing effect was observed, characterized by a decrease in MPI. A maximum projection technique was used to correct this effect and yields a more linear relationship between MPI and the contrast agent concentration. 


\section{Refill Curve and Fits}

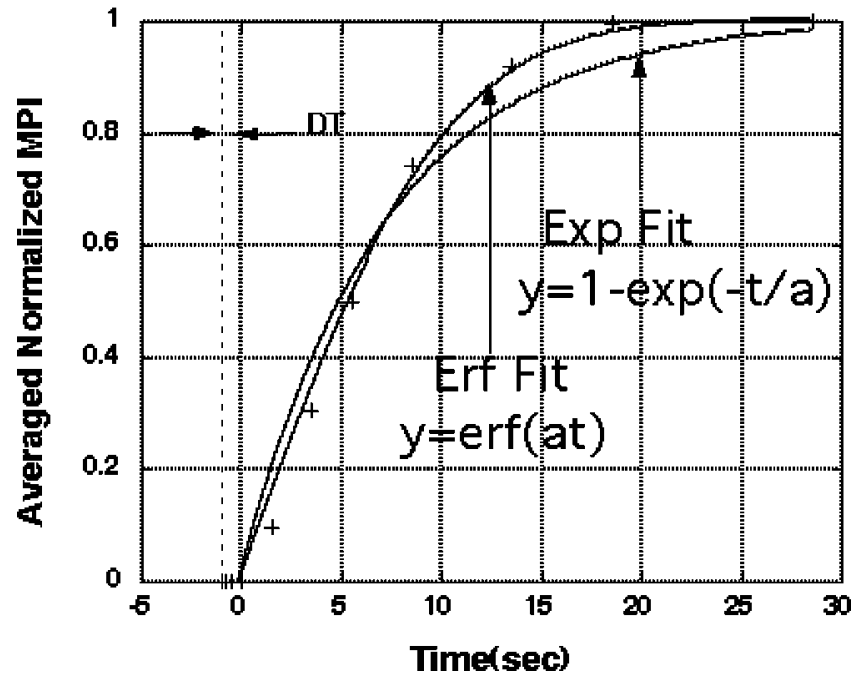

FIG. 6. A comparison between an exponential fit and an error function (erf) fit to the refill curves for the foam insert S110 and a volume flow rate of 156 $\mathrm{mL} /$ hour. For the equation $y=\operatorname{erf}(a t)$, a value of $0.12 \pm 0.01$ was found for $a$, corresponding to a MTT of $4.80 \pm 0.01 \mathrm{sec}$ while for the equation $y$ $=(1-\exp (-t / a))$, a value of $5.30 \pm 0.40 \mathrm{sec}$ was found for $a$. The delay time (DT) was equal to $1.5 \mathrm{sec}$. The time axis was shifted such that $0 \mathrm{sec}$ was defined as the time at which the MPI began to increase.

example shown in Fig. 6, the foam insert was S110 at a volume flow rate of $156 \mathrm{~mL} /$ hour. The erf function coefficient fit was $0.12 \pm 0.01$ corresponding to a MTT of $4.80 \pm 0.01 \mathrm{sec}$ while the coefficient or the MTT was 5.30 $\pm 0.40 \mathrm{sec}$ for the exponential fit. As expected, differences were observed between refill curves with different volume flow rates. However, none of the investigated properties associated with the foam inserts such as the porosity, density and echogenicity showed a consistent effect on the curves. The small differences observed between curves with identical volume flow rate was likely due to the difference in the reproducibility of experiments.

\section{MTT, bubble disruption and read-out zone estimation}

MTT can be obtained via three different methods: the two mentioned above and also via the trapezoid method based directly on the observed data points, allowing us to calculate the area above the experimental refill curves. The bubble disruption zone length ( $\mathrm{KZ}$ in $\mathrm{cm})$ as estimated from the refill measures can be calculated via the equation $\mathrm{KZ}$ $=2 \mathrm{DT} \times V+\mathrm{RO}$ where $\mathrm{RO}=\mathrm{MTT} \times V(\mathrm{~cm})$ and MTT is the mean transit time $(\mathrm{sec})$ calculated from the experimental refill curves (Table II). DT is the delay time (sec), i.e., the time for new contrast to reach the read-out zone after bubble disruption and measured from the experimental refill curves. $V$ is the linear flow velocity throughout the foam insert.

When the width of the bubble disruption zone was measured in the empty flow tube, a KZ value of $0.31 \pm 0.11 \mathrm{~cm}$ and a RO of $0.21 \pm 0.02 \mathrm{~cm}$ were found with the trapezoid method. The exponential fit gave a KZ of $0.30 \pm 0.03 \mathrm{~cm}$ with a $\mathrm{RO}$ of $0.21 \pm 0.03 \mathrm{~cm}$ and the erf fit method generated

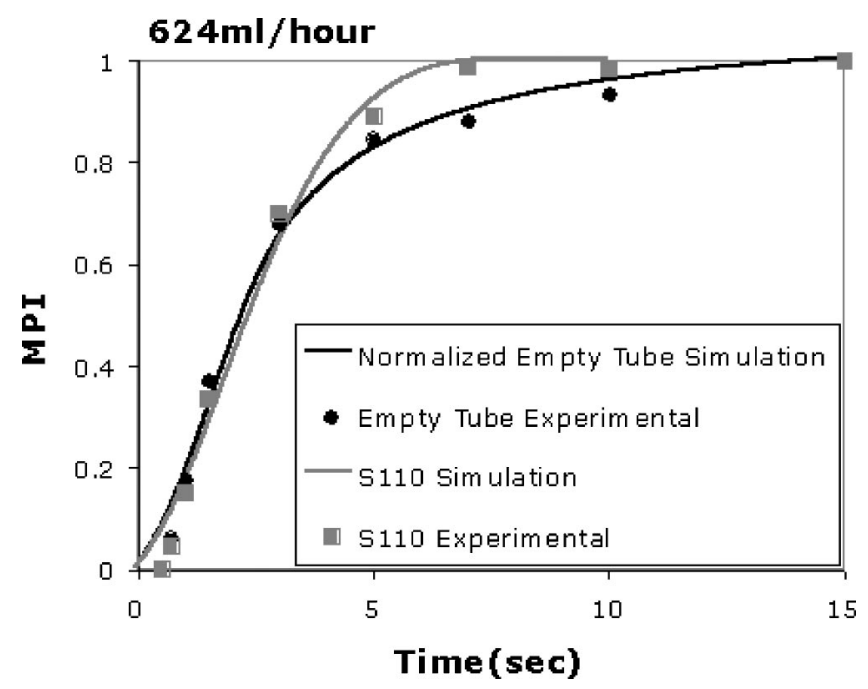

FIG. 7. Results of a simulated tube model for an empty tube and the S110 foam insert, characterized, respectively, by a parabolic and a plug flow profiles for a volume flow rate of $624 \mathrm{ml} / \mathrm{hour}$. The bubble disruption and read-out zones' width were different and both assumed to have a truncated Gaussian shape. The read-out zone was a Gaussian weighted PSF. The simulated curve for the empty tube has been normalized in the same manner as the experimental data.

a $\mathrm{KZ}$ of $0.27 \pm 0.03 \mathrm{~cm}$ with a RO of $0.18 \pm 0.03 \mathrm{~cm}$. With any of the foam inserts, the trapezoid method generated a $\mathrm{KZ}$ of $0.28 \pm 0.07 \mathrm{~cm}$ and $\mathrm{RO}$ of $0.20 \pm 0.05$, the exponential fit method gave a KZ of $0.19 \pm 0.05 \mathrm{~cm}$ and RO of 0.13 $\pm 0.03 \mathrm{~cm}$ while the erf fit method, $\mathrm{KZ}$ was $0.18 \pm 0.05 \mathrm{~cm}$ and $\mathrm{RO}$ was $0.11 \pm 0.03 \mathrm{~cm}$. These estimates are the compilation of measurements at all flow rates and demonstrate that variable flow rates still yield consistent values for $\mathrm{KZ}$ and RO widths.

To validate these results found from the experimental refill curves, a comparison between the estimated and actual bubble disruption width was needed. From Fig. 3, the shape of the bubble disruption zone can be visualized. It is seen that the zone tapers off with depth. The width of KZ ranges between 0.6 and $0.2 \mathrm{~cm}$, which is within the range of estimates listed above. However, given the variation of the KZ and $\mathrm{RO}$ with position within the tube, a better way of comparing the expected refill to the experimental results is via simulation.

\section{E. Simulation}

Using the experimental refill curves obtained for the empty tube and the foam insert S110 with a volume flow rate of $624 \mathrm{ml} /$ hour, i.e., a linear flow rate of $0.08 \mathrm{~cm} / \mathrm{sec}$ and the respective MTT (including the delay time) of 2.77 $\pm 0.02 \mathrm{sec}$ and $2.69 \pm 0.03 \mathrm{sec}$ obtained from the erf fit method, computer simulated refill curves were generated (Fig. 7).

For the simulation of the empty tube through the surrounding foam, a parabolic flow profile was assumed with a maximum velocity of $1.72 \mathrm{~mm} / \mathrm{sec}$ calculated from the known geometry of the tube and volume flow rate. The bubble disruption zone was assumed to be a Gaussian with 


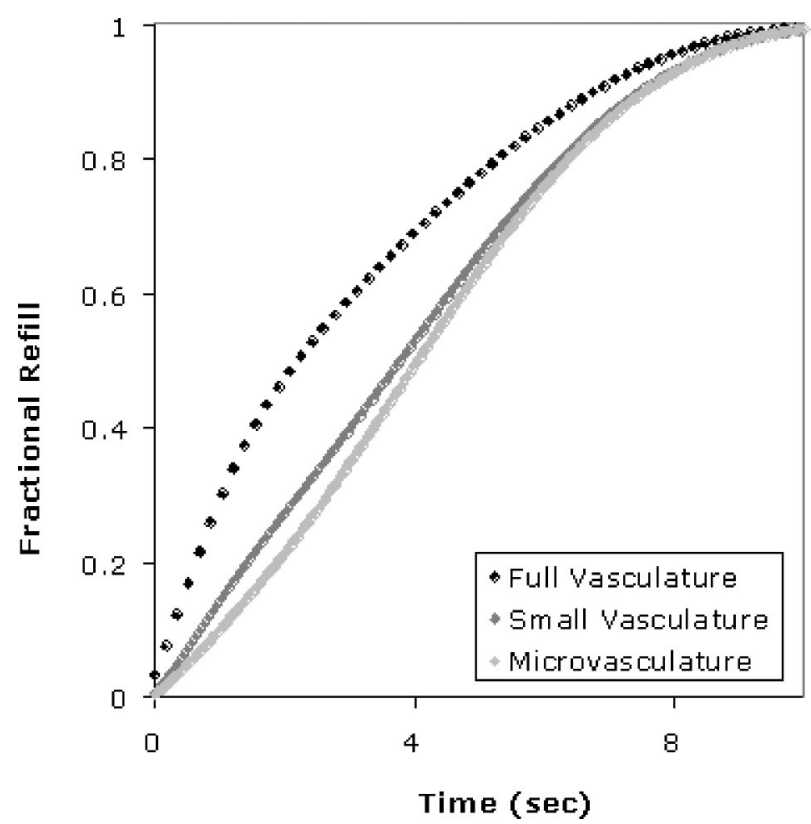

FIG. 8. Simulation of the vascular model corresponding to different velocity ranges using the serial vascular model. Broader velocity ranges display a rounder appearance than the more monotonic simulations seen when selected velocities (limited vasculature types) are used.

$\sigma=5$ and a maximum top width of $6.95 \mathrm{~mm}$ and a minimum bottom width of $1.60 \mathrm{~mm}$. These values were selected to match the observed anechoic zone (Fig. 3). The variation with depth of the read-out zone was also assumed to be a Gaussian with $\sigma=4.25$ and a maximum top width of 5.91 $\mathrm{mm}$ and a minimum bottom width of $1.36 \mathrm{~mm}$. These values were obtained from the observed anechoic zone (Fig. 3). The simulated refill curve was normalized in the same manner as the experimental data such that it resides between the $y$-ordinates 0 and 1 , yielding a simulated MTT equal to 2.78 $\mathrm{sec}$ compared to the experimental value of $2.77+/$ $-0.02 \mathrm{sec}$ obtained with the erf fit.

For the simulation of the foam insert S110, a plug or uniform flow profile of $0.8 \mathrm{~mm} / \mathrm{sec}$ was used. The bubble disruption and read-out zone characteristics were kept similar to the simulation of the empty tube. The simulated MTT was found to be $2.66 \mathrm{sec}$ compared to the experimental value of $2.69 \pm 0.03 \mathrm{sec}$ obtained using the erf fit. Both simulated and experimental curves matched very closely, not only in the MTT estimate but in shape (Fig. 7).

Vascular models based on values from Table I were generated using the serial simulation of the vascular system (Fig. 8). The full vasculature included large arteries through large veins while the small vasculature included small arteries through small veins and finally the microvasculature included the arterioles through the small veins. Therefore, different velocity ranges can be modeled resulting in differently shaped curves.

A shunting model can also be created by removing the capillaries segment from the model, so that arterioles go directly into venules. Figure 9 shows the effect of various levels of "shunting" within a normal small vasculature (from small arteries to small veins).

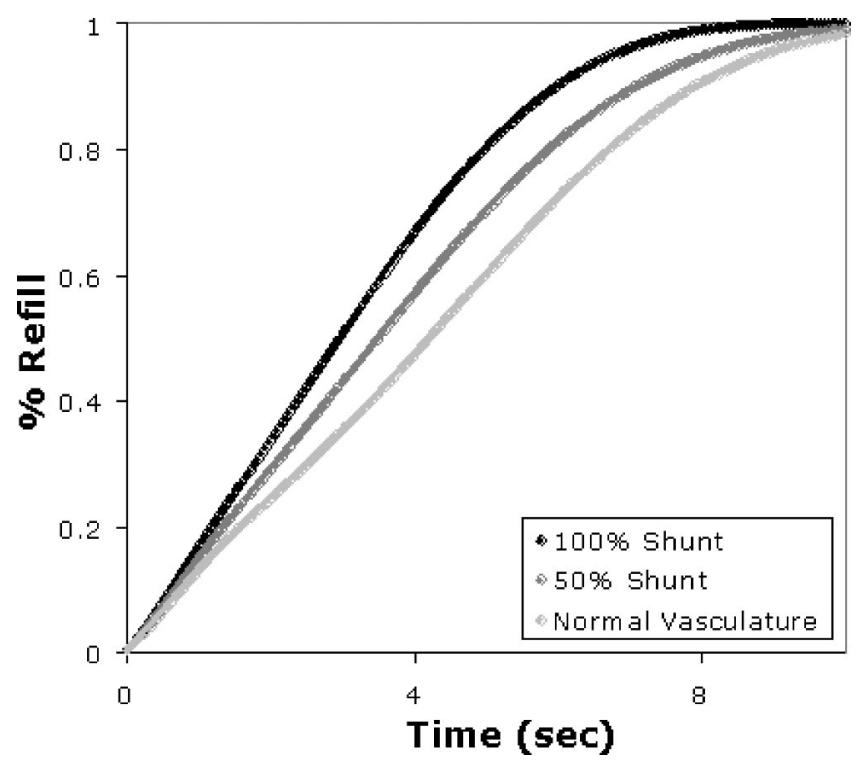

FIG. 9. A comparison between different levels of simulated shunting versus a simulated normal small vasculature. Shunting is characterized by a bypass of the capillary bed such that blood flows directly from arterioles into venules. A shunting model is therefore simulated by removing a percentage of the capillary contribution from the model.

To further investigate the potential to observe in vivo shunting using the shape of refill curves, an analysis was done on the difference between refill curves as in Fig. 9 for various percentages of shunted blood flow (Fig. 10). The peak difference of $20.3 \%$ was seen at $4.7 \mathrm{sec}$ for $100 \%$ shunting. This time corresponds to $56 \%$ refill of the normal vasculature and $77 \%$ refill of the $100 \%$ shunted blood flow. This difference scales linearly with the percentage of shunting.

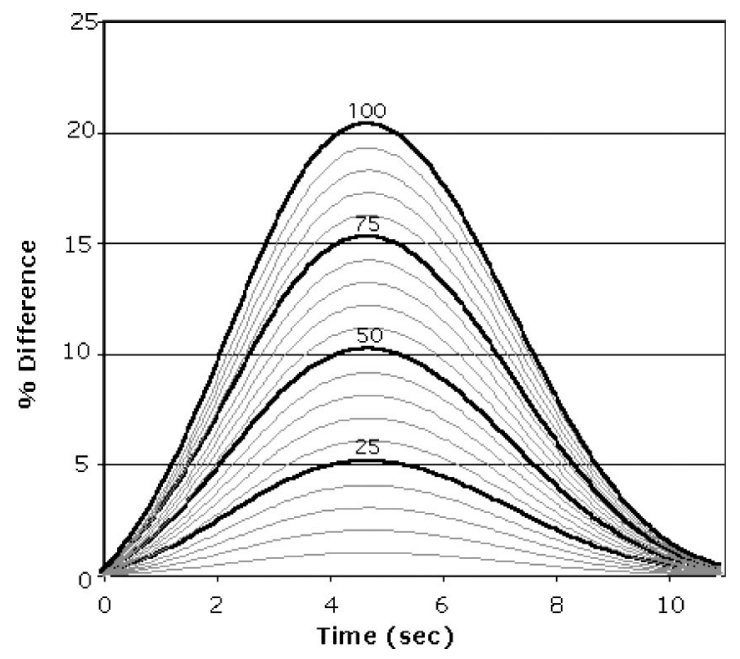

FIG. 10. The difference between normal small vasculature (as seen in Fig. 10) and shunted flow in $5 \%$ increments versus time. The maximum separation is seen at $4.7 \mathrm{sec}$ corresponding to $56 \%$ of the normal vasculature refill and $77 \%$ of the refill for the $100 \%$ shunted blood flow. For complete shunting $(100 \%)$, the maximum separation is $20.3 \%$ of the refill saturation level. This difference scales linearly with the percent shunted blood flow. 


\section{DISCUSSION}

The basic acoustic properties of the developed flow phantom proved to be reasonable for simulating in vivo tissues. The measured speed of sound for all the tested reticulated foam inserts are close to that of saline (within 1\%), reflecting the large void fraction, and about 3\% different from soft tissue. Since the void fraction of the foam is so high, one can expect to readily adjust the sound speed with temperature changes. The attenuation is somewhat higher than soft tissue but reasonable at about $0.8 \mathrm{~dB} \mathrm{~cm}^{-1} \mathrm{MHz}^{-1}$ (Table II).

The surrounding reticulated foam is used to mimic attenuation caused by overlying soft tissue in the human body. True speckle is characterized by MPI/stdev $=1$. In the reticulated foam, a value of 1.5 was observed for this ratio. This was not unexpected because the reticulated foam does not offer random scatterers but rather a somewhat regular structure. Different soft tissues display a range of values for this parameter.

The contrast agent concentration of 1/5000 used in our experiment combined with the high MI FEI technique resulted in a veil effect. This problem was overcome by using the maximum projection technique for the refill curve analysis (Fig. 5). With this technique, a linear relationship between MPI and contrast agent concentration was observed over the wide range of concentrations.

In the foam insert experiments, the error function (erf) fit offers better smoothing for the experimental refill curves than the exponential fit assumed by Wei et al. ${ }^{3,4}$ (Fig. 6). This cannot be assumed to apply to all velocity profiles because there is no physical rationale behind its selection. It is also important to take into account the delay time (DT) between the bubble disruption event and the time when the actual refill begins. A previous study done by Lucidarme et al. ${ }^{10}$ also found a sigmoid shape function to be a better fit to the refill curve. Their finding was for different reasons, however, as their experimental setup employed a different imaging plane with continuous low MI imaging.

The width of both the bubble disruption (KZ) and readout (RO) zones varies with depth and can be approximated by a truncated Gaussian as seen in Fig. 3. This shape is likely due to attenuation from the contrast agent and/or the foam. It is also likely to depend on the number of high MI pulses constituting a pulse sequence. Therefore, experimental variability can be introduced by effects on both transmitted and received pulses. This is of importance because the shape of these zones affect the shape of the refill curves. If both zones were square in shape, the curvature found in the refill curves would then be due only to the range of velocities and the read-out zone's PSF.

The imaging system used for the experiments has limited suppression of a nonbubble background signal. Because of the high echogenicity of the foam insert, the resulting images suffer from lack of dynamic range. This can be overcome by using a newer imaging system which incorporates better background suppression.

In general, the refill curves were affected by the volume flow rate (or linear velocity) of the fluid traversing the foam inserts but not by the density or porosity of the insert. This was not unexpected, because the high void volume of the foam does not divert the primary axial flow, but rather serves to effectively segment the flow into many small channels. As a result, the major effect of the foam insert was probably to alter the parabolic flow profile of an empty tube to something close to the uniform velocity profile of plug flow.

From the tube simulation, a noticeable difference was observed in the shape of the refill curves in the case of a parabolic versus plug flow profile (Fig. 7). This difference was mainly observed at the high end of the refill curve and compares well with the experimental data. While the MTT is comparable, total refill was much slower and the curve has a rounder shape for the parabolic flow profile compared to the presumed plug flow profile through the foam.

In the serial vascular system simulations, it can be concluded that the range of velocities also affects the shape of the refill curves. Wider velocity ranges found in the full vasculature which includes large arteries through large veins produce a rounder refill curve shape (Fig. 8). A flatter refill curve shape was generated when the large vessels such as large arteries and large veins were removed from the simulation. The shape of the refill curve gets even flatter for the microvasculature simulation, i.e., when the only vessels present in the model were arterioles, capillaries and venules.

When "shunting" was simulated, the refill occurred sooner due to the absence of capillaries in the model (Fig. 9). The maximum separation between curves of varying shunt percentage appears to occur at around the $56 \%$ refill level of the normal vasculature.

The simulations also indicate that the best time to detect changes in the refill curves due to shunting is $4.7 \mathrm{sec}$ into the refill. In order to detect shunting below $5 \%$, the error in this refill measurement should be less than $1 \%$. These preliminary simulations show some intriguing results based on relatively simple but relevant changes in the vascular structure.

When observing the shape of the refill curves, it is important to take into account the shape of both the receiving beam profile and the bubble disruption profile. Simulations show that refill curves can be used for more than the mean transit time estimation. Information about velocity profiles and even abnormal vascularity such as shunting can also be detected.

It is interesting to note that a previous study ${ }^{11}$ found that the time to the $80 \%$ contrast replenishment level was a robust parameter for distinguishing normal from cancerous blood flow in a rat. Our simulation techniques should accommodate more refined vascular models and make it possible to test hypotheses that might explain this and other phenomenon related to refill curves and abnormal vasculature.

\section{ACKNOWLEDGMENTS}

This work was supported in part by PHS Grant No. R01 DK56658 and NIST Cooperative Agreement No. 2001-004392. Equipment support was provided by Toshiba Medical Systems. 


\section{APPENDIX: AREA ABOVE AN ERROR FUNCTION}

Knowing that

$$
\int \operatorname{erf}(x) d x=x \operatorname{erf}(x)+\frac{e^{-x^{2}}}{\sqrt{\pi}} .
$$

Let $u=a x$, so that $d u=a d x$ :

$$
\begin{aligned}
\int_{0}^{\infty}(1-\operatorname{erf}(a x)) d x & =\frac{1}{a}\left(\int_{0}^{\infty}(1-\operatorname{erf}(u)) d u\right) \\
& =\frac{1}{a}\left[u-\operatorname{erf}(u)+\frac{e^{-u^{2}}}{\sqrt{\pi}}\right]_{0}^{\infty} \\
& =\left[x(1-\operatorname{erf}(a x))+\frac{e^{-(a x)^{2}}}{a \sqrt{\pi}}\right]_{0}^{\infty} \\
& \rightarrow \frac{1}{a \sqrt{\pi}} .
\end{aligned}
$$

Thus the area above the error function is $1 / a \sqrt{\pi}$.

\footnotetext{
${ }^{a)}$ Electronic mail: titainap@umich.edu

${ }^{1}$ W. F. Hamilton, J. W. Moore, J. M. Kinsman, and R. G. Spurling, "Further analysis of the injection method, and of changes in hemodynamics under physiological and pathological conditions," Am. J. Phys. 99, 534551 (1932).

${ }^{2}$ P. Meier and K. L. Zierler, "On the theory of the indicator-dilution method for measurement of blood flow and volume," J. Appl. Phys. 6, 731-744 (1954).

${ }^{3}$ K. Wei, D. M. Skyba, C. Firschke, A. R. Jayaweera, J. R. Lindner, and S.
}

Kaul, "Interactions between microbubbles and ultrasound: in vitro and in vivo observations," J. Am. Coll. Cardiol. 29, 1081-1088 (1997).

${ }^{4}$ K. Wei, A. R. Jayaweera, S. Firoozan, A. Linka, D. M. Skyba, and S. Kaul, "Quantification of myocardial blood flow with ultrasound-induced destruction of microbubbles administred as a constant venous infusion," Circulation 97, 473-483 (1998).

${ }^{5}$ N. Kamiyama, F. Moriyasu, Y. Kono, Y. Mine, T. Nada, and B. Yamasaki, "Investigation of the 'flash echo' signal associated with a US contrast agent," Radiology 201, 165 (1996).

${ }^{6}$ F. Moriyasu, Y. Kono, T. Nada, T. Matsumura, Y. Suginoshita, K. Kobayashi, "Flash echo (passive cavitation) imaging of the liver by using US contrast agents and intermittent scanning sequence," Radiology 201, 374 (1996).

${ }^{7}$ K. Ohmori, B. Cotter, O. L. Kwan, K. Mizushige, and A. DeMaria, "Relation of contrast echo intensity and flow velocity to the amplification of contrast opacification produced by intermittent ultrasound transmission," Am. Heart J. 134, 1066-1074 (1997).

${ }^{8}$ S. Wilson, P. Burns, R. Muradali, J. Wilson, and X. Lai, "Harmonic hepatic US with microbubble contrast agent: initial experience showing improved characterization of hemangioma, hepatocellular carcinoma, and metastasis," Radiology 215, 153-161 (2000).

${ }^{9}$ G. L. LeCarpentier, N. G. Chen, J. B. Fowlkes, and P. L. Carson, "Preliminary results of a new method of 3D ultrasound contrast agent mapping of vascular anomalies for characterization of breast cancer," Era of Hope Department of Defense Breast Cancer Research Program, 25-28 September, Orlando, Proceedings 29-6, 2002.

${ }^{10}$ O. Lucidarme, S. Franchi-Abella, J. M. Correas, S. Bridal, E. Kurtisovski, and G. Berger, "Blood flow quantification with contrast-enhanced US: 'Entrance in the section' phenomenon-Phantom and Rabbit study," Radiology 228, 473-479 (2003).

${ }^{11}$ R. E. Pollard, A. R. Sadlowski, S. H. Bloch, L. Murray, E. R. Wisner, S. Griffey, and K. W. Ferrara, "Contrast-assisted destruction-replenishment ultrasound for the assessment of tumor microvasculature in a rat model," Technol. Cancer Res. Treat. 6, 459-470 (2002).

${ }^{12} \mathrm{http}: / /$ www.mas.ncl.ac.uk/ sbrooks/book/nish.mit.edu/2006/Textbook/ Nodes/chap09/node22.html. 